\title{
BOUNDED HOLOMORPHIC FUNCTIONS OF SEVERAL COMPLEX VARIABLES. I
}

\author{
BY \\ DONG SIE KIM( $\left.{ }^{1}\right)$
}

\begin{abstract}
A domain of bounded holomorphy in a complex analytic manifold is a maximal domain for which every bounded holomorphic function has a bounded analytic continuation. In this paper, we show that this is a local property: if, for each boundary point of a domain, there exists a bounded holomorphic function which cannot be continued to any neighborhood of the point, then there exists a single bounded holomorphic function which cannot be continued to any neighborhood of the boundary points.
\end{abstract}

Introduction.. Let $X$ be a topological space. A subset $D$ of $X$ is said to be a region if it is open and $i \hat{i}$ is said to be a domain if it is open and connected. We denote by $N(p)$ a fundamental system of open neighborhoods of $p$, where $p \in X$.

1. Definition. Let $X$ be a topological space and $U$ be an open subset of $X$. Let $C(U)$ be the family of all continuous complex-valued functions on $U$, then $C(U)$ is an algebra with 1 , and it is equipped with the topology of uniform convergence on compact subsets of $U$. For a pair of open subsets $U$ and $V$ in $X$ such that $V \subset U$ we define $\pi_{U V}: C(U) \rightarrow C(V)$ by $\pi_{U V} f=f \mid V$. Let $A(U)$ be a subalgebra of $C(U)$ with 1 and we assume that $\pi_{U V} A(U) \subset A(V)$; then we call $A=\left\{A(U), \pi_{U V}\right\}$ a presheaf of algebras of functions. A presheaf $A$ has the local belonging property if, for all open sets $U$ of $X$ and $f$ in $C(U)$, for every $p \in U$ there is $V \in N(p), V \subset U$, such that $f \mid V \in A(v)$; then $f \in A(U)$.

A sheaf $A$ of algebras of functions is a presheaf of algebras of functions with the local belonging property. $A$ is said to be a ringed structure on $X$ and the pair ( $X, A)$ is said to be a ringed space. The functions in $A(U)$ are $A$-holomorphic functions. We note that $A(U)$ has the relative topology induced by the topology on $C(U)$.

A ringed structure $A$ on $X$ is an $n$-dimensional complex analytic structure on $X$ if for all $x \in X$ there are $U \in N(x)$ and $f_{1}, \ldots, f_{n} \in A(U)$ such that

$$
F=\left(f_{1}, \ldots, f_{n}\right): U \rightarrow C^{n}
$$

Received by the editors February 5, 1970 and, in revised form, September 16, 1970.

AMS 1970 subject classifications. Primary 32D15; Secondary 32D05.

Key words and phrases. Ringed space, complex analytic manifold, quasi-analytic, hausdorff sheaf, analytic extension, analytic continuation, montel property, weak region of bounded holomorphy, region of bounded holomorphy, spectrum.

( ${ }^{1}$ This paper is a portion of the author's dissertation written under the supervision of Professor Frank D. Quigley at Tulane University. 
is a homeomorphism of $U$ onto $F(U)$ with the properties: $F(U)$ is open in $C^{n}$ and for all $W$ open $\subset U,\left\{f \circ(F \mid W)^{-1}: f \in A(W)\right\}=\mathcal{O}(F(W))$, where $\mathcal{O}$ is a complex analytic structure on $C^{n}$. If $X$ is a hausdorff space we call this pair $(X, A)$ a complex analytic manifold.

For a subset $U$ of $X, A(U)$ is quasi-analytic if for all nonempty open subsets $V$ of $U$ and for $f, g$ in $A(U)$ such that $f=g$ on $V$ then $f=g$ on $U$.

We give a characterization of quasi-analyticity in terms of the hausdorffness of the topology on $A$ in the following proposition. The proof may be found in (3).

2. Proposition. Let $(X, A)$ be a ringed space with $X$ a locally connected hausdorff space. Then $A$ is hausdorff if and only if $A(U)$ is quasi-analytic for all connected subsets $U$ of $X$.

\section{Regions of bounded holomorphy.}

3. Definition. Let $(X, A)$ be a ringed space and $D$ be a region. We define $B(D)$ $=\{f \in A(D): f$ is bounded on $D\}$. For a point $p \in \bar{D}-D$ (boundary of $D$ ) and $U \in N(p)$, a function $f \in B(D)$ is said to be extendable to $U$ if there is a function $g \in B(U)$ such that $f=g$ on $D \cap U . D$ is said to be a weak region of bounded holomorphy if there exists a function $f \in B(D)$ which cannot be extendable beyond the boundary of $D$.

$A$ is said to be montel if for an open set $U$ in $X$ and $F \subset A(U)$ there is $M_{K}>0$ such that $\|f\|_{K}<M_{K}$ for all $f \in F$ and for all compact subsets $K$ of $U$; then $F$ is relatively compact in $A(U)$.

$A$ is c.o. complete if for all open subsets $U$ in $X, A(U)$ is complete in the topology of uniform convergence on compact subsets of $U$.

We note that an analytic structure $A$ in a complex analytic manifold $(X, A)$ has the montel property, and it is hausdorff and c.o. complete.

We show that the weak bounded holomorphy is a local property in the following theorem.

4. Lemma. Let $(X, A)$ be a ringed space. We assume that $X$ is a locally compact and locally connected hausdorff space, and $A$ is hausdorff, c.o. complete and montel. Let $D$ be a region in $X$ and $p \in \bar{D}-D$ such that $X$ is first countable at $p$. Let $B$ be $a$ closed (relative to the topology of uniform convergence on $D$ ) subalgebra of $B(D)$. Then these are equivalent:

$\left(1^{\circ}\right)$ For every $U_{\alpha} \in N(p)$ there is a function $f_{\alpha} \in B$ which cannot be extended to $U$.

$\left(2^{\circ}\right)$ There is a function $f \in B$ which cannot be extended to any neighborhood of $P$.

Proof. It is sufficient to show that $\left(1^{\circ}\right)$ implies $\left(2^{\circ}\right)$. Let $\left\{U_{m}: m \in Z_{+}\right\}$be a countable nested basis of open neighborhood of $p$. Let $B_{1}\left(U_{m}, n\right)=\{f \in B: f=g \mid D$ where $g \in B\left(D \cup U_{m}\right)$ and $\left.\|g\|_{U_{m}} \leqq n\right\}, n \in Z_{+}$. We claim that $B_{1}\left(U_{m}, n\right)$ is a closed nowhere dense subset of $B$. For closedness, let $\left\{f_{k}\right\}$ be any net in $B_{1}\left(U_{m}, n\right)$ converging uniformly on $D$ to $f$. We note that $\left\{f_{k}\right\}$ is c.o. convergent to $f$. Let $\left\{g_{k}\right\}$ $\subset B\left(D \cup U_{m}\right)$ such that $g_{k} \mid D=f_{k},\left\|g_{k}\right\|_{U_{m}} \leqq n, k \in Z_{+} \cdot\left\{g_{k}\right\}$ is uniformly bounded on 
compact subsets of $D \cup U_{m}$. Since $A$ is montel $\left\{g_{k}\right\}$ is relatively compact in $A\left(D \cup U_{m}\right)$. Thus there is a subnet $\left\{g_{i}\right\} \subset\left\{g_{k}\right\}$ which converges to $g \in A\left(D \cup U_{m}\right)$. Now $\lim _{\text {c.o. }} g_{j} \mid D=\lim _{\text {c.o. }} f_{j}=f$, so $g \mid D=f$ and since $\left\|g_{j}\right\|_{U_{m}} \leqq n$ for $j \in Z_{+},\|g\|_{U_{m}} \leqq n$, which concludes that $f \in B_{1}\left(U_{m}, n\right)$. For nowhere denseness, let $B_{1}\left(U_{m}, n\right)$ $=\bigcup_{n} B_{1}\left(U_{m}, n\right)$. Take $f \in B-B_{1}\left(U_{m}\right)$ and define $g_{j}=j^{-1} f+h$ for $h \in B_{1}\left(U_{m}, n\right)$, $j \in Z_{+}$. Then $g_{j} \notin B_{1}\left(U_{m}\right)=B_{1}\left(U_{m}, n\right)$ and $\lim _{j} g_{j}=h$. Since $h$ is an arbitrary element of $B_{1}\left(U_{m}, n\right)$, int $B_{1}\left(U_{m}, n\right)=\varnothing$.

Let $B_{1}=\bigcup\left\{B_{1}\left(U_{m}\right): m \in Z_{+}\right\}$and $B_{2}=\{f \in B: f$ can be extended to some neighborhood of $p\}$. Then $B_{1}=B_{2}$. Now since $B$ has the baire property, $B_{1} \subsetneq B$. Hence there is $f \in B-B_{1}$, so $f \notin B_{2}, f$ cannot be extended to any neighborhood of $p$.

5. THeOREM. Let $(X, A)$ be a ringed space. We assume that $X$ is a locally compact locally connected hausdorff space, and $A$ is hausdorff, c.o. complete and montel. Let $D$ be a region in $X$ such that $\bar{D}-D$ is separable and $X$ is first countable on $\bar{D}-D$. Let $B$ be a closed subalgebra of $B(D)$ as in the lemma. Then these are equivalent:

$\left(1^{\circ}\right)$ For every $p \in \bar{D}-D$ there is a function $f_{p} \in B$ which cannot be extended to any $U \in N_{(p)}$.

$\left(2^{\circ}\right)$ There is a function $f \in B$ which cannot be extended beyond the boundary of $D$.

Proof. Let $\left\{U_{m}: m \in Z_{+}\right\}$be a countable basis of nested open neighborhoods of $p \in \bar{D}-D$. Let $B_{1}\left(p, U_{m}, n\right)=\left\{f \in B: f=g \mid D, g \in B\left(D \cup U_{m}\right),\|g\|_{U_{m}} \leqq n\right\}, n$, $m \in Z_{+}$. Then $B_{1}\left(p, U_{m}, n\right)$ is a closed nowhere dense subset of $B$ as in the proof of the lemma. Let $\left\{p_{i}: i \in Z_{+}\right\}$be a countable dense subset of $\bar{D}-D$ and $\left\{U_{m}^{(i)}\right\}$ be a countable basis of nested open neighborhoods of $p_{i}$. Let

$$
B_{2}=\bigcup\left\{B_{1}\left(p_{i}, U^{(i)}, n\right): i, m, n \in Z_{+}\right\}
$$

and

$$
B_{3}=\{f \in B: f \text { can be extended beyond } \bar{D}-D\} \text {. }
$$

Then $B_{2}=B_{3}$. Since $B$ is baire, $B_{2} \varsubsetneqq B$. Hence there is $f \in B-B_{2}=B-B_{3}$, which asserts $\left(2^{\circ}\right)$.

6. Corollary. Let $(X, A)$ be a complex analytic manifold and $D$ be a region in $X$ such that $\bar{D}-D$ is separable and $X$ is first countable on $\bar{D}-D$. Let $B=B(D)$. Then these are equivalent:

$\left(1^{\circ}\right)$ For every $p \in \bar{D}-D$ there is an $f \in B$ which cannot be extended to any $U \in N(p)$.

$\left(2^{\circ}\right) D$ is a weak region of bounded holomorphy.

7. Definition. Let $(X, A)$ be a ringed space and $D$ be a region in $X$. Let $V$ be an open subset of $X$ such that $D \cap V \neq \varnothing$ and $V \notin D . f \in B(D)$ is said to be continued to $V$ if there is a connected component $\Omega$ of $D \cap V$ and $g \in B(V)$ such that $f=g$ on $\Omega$. We say that $g$ is a continuation of $f$ to $V$. A boundary point $p$ of $D$ is said to be a boundary singularity for $f \in B(D)$ if $f$ cannot be continued to any open 
neighborhood of $p$. A region is called a region of bounded holomorphy if there is an $f \in B(D)$ for which every boundary point of $D$ is a boundary singularity.

We give a characterization of a region of bounded holomorphy by a local property in the next theorem.

8. Lemma. Let $(X, A)$ be a ringed space. We assume that $X$ is a locally compact and locally connected hausdorff space and $A$ is hausdorff, c.o. complete, and montel. Let $D$ be a region in $X$ and $p \in \bar{D}-D$ such that $X$ is first countable at $p$. Let $B$ be a closed (relative to the topology of uniform convergence on $D$ ) subalgebra of $B(D)$. Then these are equivalent:

$\left(1^{\circ}\right)$ For every $U_{\alpha} \in N(p)$ and every connected component $\Omega_{\alpha \beta}$ of $U_{\alpha} \cap D$ there is $f_{\alpha \beta} \in B$ such that $f_{\alpha \beta}$ has no continuation to $U_{\alpha}$.

$\left(2^{\circ}\right)$ There is $f \in B$ such that for all $U \in N(p)$ and for all connected components $\Omega$ of $U \cap D, f$ has no continuation to $U_{\alpha}$, i.e. $p$ is a boundary singularity for $f$.

Proof. It suffices to show that $\left(1^{\circ}\right)$ implies $\left(2^{\circ}\right)$. Let $\left\{U_{\alpha}: \alpha \in Z_{+}\right\}$be a countable nested basis of open neighborhoods of $p$ and let $\left\{\Omega_{\alpha \beta}: \beta \in Z_{+}\right\}$be a countable family of connected components of $U_{\alpha} \cap D$. Let $B_{1}\left(\Omega_{\alpha \beta}, n\right)=\{f \in B$ : there is $g \in B\left(U_{\alpha}\right)$ such that $f=g$ on $\Omega_{\alpha \beta}$ and $\left.\|g\|_{U_{\alpha}} \leqq n\right\}, n \in Z_{+}$. Then as in the proof of Lemma $4, B_{1}\left(\Omega_{\alpha \beta}, n\right)$ is a closed nowhere dense subset of $B$. Let $B_{1}=\bigcup_{\alpha, \beta, n} B_{1}\left(\Omega_{\alpha \beta}, n\right)$ and let $B_{2}=\{f \in B: f$ can be continued to some neighborhood of $p\}$. Then $B_{1}=B_{2}$, and since $B_{1} \varsubsetneqq B$ there is an $f \in B-B_{2}$.

9. THEOREM. Let $(X, A)$ be a ringed space. We assume that $X$ is a locally compact, locally connected hausdorff space and $A$ is hausdorff, c.o. complete and montel. Let $D$ be a region in $X$ such that $\bar{D}-D$ is separable and $X$ is first countable on $\bar{D}-D$. Let $B$ be a closed subalgebra of $B(D)$. Then these are equivalent:

$\left(1^{\circ}\right)$ For every $p \in \bar{D}-D$ there is a function $f_{p} \in B$ for which $p$ is a boundary singularity.

$\left(2^{\circ}\right)$ There is a function $f \in B$ for which every boundary point is a boundary singularity.

Proof follows by the lemma and in a similar way as the proof of Theorem 5.

10. Corollary. Let $(X, A)$ be a complex analytic manifold and $D$ be a region in $X$ such that $\bar{D}-D$ is separable. Let $B=B(D)$. Then these are equivalent:

$\left(1^{\circ}\right)$ For every $p \in \bar{D}-D$ there is $f_{p} \in B$ for which $p$ is a boundary singularity.

$\left(2^{\circ}\right) D$ is a region of bounded holomorphy.

In the following, we show that a weak region of bounded holomorphy is a region of bounded holomorphy when the region is locally connected on the boundary.

11. Definition. Let $X$ be a topological space and $D$ be a region in $X$. We say that $D$ is locally connected at $p \in \bar{D}-D$ if $p$ has a base of open neighborhoods whose intersections with $D$ are connected. $D$ is locally connected on the boundary of $D$ if $D$ is locally connected at every point of the boundary.

The following lemma will give the proof of Theorem 13. 
12. LemMA. Let $X$ be a locally connected hausdorff space and let $D$ be a region in $X$ which is locally connected on the boundary. Let $V \in N(p), p \in \bar{D}-D$ and $U$ be an open subset of $V \cap D$. Then there is an open set $V_{1} \subset U$ such that $V_{1} \cap(\bar{D}-D)$ $\neq \varnothing, V_{1} \cap D$ is connected and $V_{1} \cap U \neq \varnothing$.

Proof. We assume that $V$ is a connected neighborhood of $p$.

(i) We show that for every connected component $\Omega$ of $V \cap D, V \cap(\bar{\Omega}-\Omega)$ $\subset \bar{D}-D$. Note that $V \cap(\bar{\Omega}-\Omega) \neq \varnothing$, for otherwise we have $V=(V-\bar{\Omega}) \cup \Omega$ which contradicts its connectedness. Now $\bar{\Omega} \subset \bar{D}$ so that $V \cap(\bar{\Omega}-\Omega) \subset V \cap \bar{D}$. If $V \cap(\bar{\Omega}-\Omega) \cap D \neq \varnothing$, take $p \in V \cap(\bar{\Omega}-\Omega) \cap D$ then there is a connected open set $U^{\prime} \in N(p)$ such that $U^{\prime} \subset V \cap D$ and $U^{\prime} \cap \Omega \neq \varnothing$. Thus $U^{\prime} \cup \Omega \subset V \cap D$ is connected. But then $U^{\prime} \cup \Omega=\Omega$ and $p \in \Omega$, which is a contradiction. It follows that $V \cap(\bar{\Omega}-\Omega) \cap D=\varnothing$ so that $V \cap(\bar{\Omega}-\Omega) \subset \bar{D}-D$.

(ii) Choose a connected component $\Omega$ of $V \cap D$ such that $\Omega \cap U \neq \varnothing$. Take $q \in V \cap(\bar{\Omega}-\Omega) \subset \bar{D}-D$ and choose a neighborhood $V^{\prime}$ of $q$ such that $V^{\prime} \subset V$ and $V^{\prime} \cap D$ is connected. Let $V_{1}=\Omega \cup V^{\prime}$. Since $\Omega \cap V^{\prime} \neq \varnothing, V_{1}$ has the required property.

13. TheOREM. Let $(X, A)$ be a ringed space. We assume that $X$ is a locally compact, locally connected hausdorff space, and $A$ is hausdorff, c.o. complete and montel. Let $D$ be a region in $X$ which is locally connected on the boundary. Let $B$ be a closed subalgebra of $B(D)$. Then these are equivalent:

$\left(1^{\circ}\right)$ There is a function $f \in B$ which cannot be extended beyond $D$.

$\left(2^{\circ}\right)$ There is a function $f \in B$ which cannot be continued beyond $D$.

Proof. It is immediate from the lemma.

14. Corollary. Let $(X, A)$ be a complex analytic manifold. Let $D$ be a region which is locally connected on the boundary. Then these are equivalent:

$\left(1^{\circ}\right) D$ is a weak region of bounded holomorphy.

$\left(2^{\circ}\right) D$ is a region of bounded holomorphy.

We investigate regions of bounded holomorphy in $\left(C^{n}, \mathcal{O}\right)$. First, we have a useful lemma for searching domains of bounded holomorphy.

15. Lemma. Let $(X, A)$ be a complex analytic manifold and $D$ be a region in $X$. Let $U$ be a domain such that $D \cap U \neq \varnothing$ and $U \notin D$. If every function $f \in B(D)$ can be continued to $U$ and $\tilde{f}$ denotes the continuation of $f$ to $U$, then $\tilde{f}(U) \subset \mathrm{cl}(f(D))$ for all $f \in B(D)$.

Proof. Let $\alpha \notin \mathrm{cl}(f(D))$, then $g=(f-\alpha)^{-1} \in B(D)$, and so has a continuation $\tilde{g} \in B(U)$. Now $g \cdot(f-\alpha) \equiv 1$ on $D$, and $g \cdot(f-\alpha)=\tilde{g} \cdot(\tilde{f}-\alpha)=1$ on a connected component $\Omega$ of $D \cap U$. So by analytic continuation, $\tilde{g} \cdot(\tilde{f}-\alpha) \equiv 1$ on $U$. Hence $\alpha \notin \tilde{f}(U)$. So $\tilde{f}(U) \subset \mathrm{cl}(f(D))$.

16. Simple examples of domains of bounded holomorphy in $\left(C^{n}, \mathcal{O}\right)$.

$\left(1^{\circ}\right)$ An open polydisc

$\boldsymbol{P}(w: r)=\boldsymbol{P}\left(w_{1}, \ldots, w_{n}: r_{1}, \ldots, r_{n}\right)=\left\{s \in \boldsymbol{C}^{n}:\left|s_{i}-w_{i}\right|<r_{i}, 1 \leqq i \leqq n\right\} \subset \boldsymbol{C}^{n}$ 
is a domain of bounded holomorphy. For, take a boundary point $s \in \overline{\boldsymbol{P}}(w: r)$; then $\left|s_{j}\right|=r_{j}$ for some $j$. Now for any polydisc $\boldsymbol{P}_{1}(s: \varepsilon),\left\|z_{j}\right\|_{\boldsymbol{P}_{1}}>\boldsymbol{r}_{j}$. Hence $z_{j}(\boldsymbol{P})$ $\$ \mathrm{cl}\left(Z_{j}(\boldsymbol{P})\right)$. By Lemma $15, \boldsymbol{P}$ is a domain of bounded holomorphy. Moreover, an analytic polyhedron and a bounded complete Reinhardt domain are domains of bounded holomorphy.

$\left(2^{\circ}\right)$ A simply connected domain $D$ in $C$ which is locally connected on the boundary of $D$ is a domain of bounded holomorphy.

17. Proposition. Let $\left\{D_{j}: j \in Z_{+}\right\}$be an indexed set of regions of bounded holomorphy in $C^{n}$. Let $D=\bigcap_{j=1}^{\infty} D_{j}$ and assume that $D$ is open. Then $D$ is a region of bounded holomorphy in $\boldsymbol{C}^{n}$.

Proof. For a point $p \in \bar{D}-D$ there exists $m \in Z_{+}$such that $p \notin D_{m}$. Then there exists $f \in B\left(D_{m}\right)$ which is a singular function at $p$. Thus $f \mid D \in B(D)$ is singular at $p$.

18. Proposition. A finite cartesian product of regions of bounded holomorphy is a region of bounded holomorphy.

Proof. We shall prove this for the case of a product of two regions. Let $D_{1}$ and $D_{2}$ be regions of bounded holomorphy in $C^{n}$ and let $f_{i} \in B\left(D_{\imath}\right), i=1,2$, be singular functions. Define $F_{1} \in B\left(D \times C^{n}\right)$ by $F_{1}(s, t)=f_{1}(s)$ and $F_{2} \in B\left(C^{n} \times D_{2}\right)$ by $F_{2}(s, t)$ $=f_{2}(t)$. Then $F_{1}$ is a singular function at every point of (bdry $\left.D_{1}\right) \times C^{n}$ and so is $F_{2}$ for $C^{n} \times\left(\right.$ bdry $\left.D_{2}\right)$. For, if $F_{1}$ is not, then there is $V \in N(p), p \in\left(\right.$ bdry $\left.D_{1}\right) \times C^{n}$ such that $F_{1}$ can be continued to $V$. Let $W$ be the image of $V$ into $C^{n} \supset D_{1}$ then $F_{1} \mid W=f_{1}$ can be continued to $W$. But $W$ is a neighborhood of a boundary point of $D_{1}$. This is absurd (similarly for $\left.F_{2}\right)$. Now bdry $\left(D_{1} \times D_{2}\right)=\left(\right.$ bdry $\left.D_{1}\right) \times \bar{D}_{2}$ $\cup \bar{D}_{1} \times\left(\right.$ bdry $\left.D_{2}\right)$. Thus if $p \in$ bdry $\left(D_{1} \times D_{2}\right)$, then $F_{1}$ or $F_{2}$ is a singular function at $p$. Hence $D_{1} \times D_{2}$ is a domain of bounded holomorphy.

19. Proposition. Every convex (in the geometric sense) domain $D$ in $C^{n}$ is a domain of bounded holomorphy.

Proof. Since such a domain $D$ is the intersection of the open halfspaces in $C^{n}$ (as a real vector space $R^{2 n}$ ) which contain it, by Proposition 17 it suffices to show that every open halfspace in $\boldsymbol{C}^{n}$ is a domain of bounded holomorphy. Let $S=\left\{\left(z_{1}, \ldots, z_{n}\right) \in C^{n}: \operatorname{Re} z_{i}>0, i=1, \ldots, n\right\}$. Then any open halfspace in $C^{n}$ can be identified as $S$ by a translation and a complex linear transformation. Hence again it suffices to show that $S$ is a domain of bounded holomorphy. But this is so; for, let $H=\{z \in C: \operatorname{Re} z>0\}$, then since $H$ can be mapped onto the open unit disc by a Riemann map, $H$ is a domain of bounded holomorphy. Now $S=\prod^{n} H$, a finite cartesian product. Hence $S$ is a domain of bounded holomorphy by Proposition 18.

20. Proposition. Let $D$ be a region in $C^{n}, n>1$, and let $K$ be a compact subset of $D$ such that $D-K$ is connected. Then for every $f \in B(D-K)$ there exists $\tilde{f} \in B(D)$ such that $f=\tilde{f}$ on $D-K$. 
Proof. Since $B(D-K) \subset \mathcal{O}(D-K)$, for every function $f \in B(D-K)$ there is $\tilde{f} \in \mathcal{O}(D)$ such that $f=\tilde{f}$ on $D-K$ by a theorem of Hartog's. So it suffices to show that those extensions are still bounded on $D$. But this is clear from Lemma 15.

21. Let $D$ be a region in $C^{n}$ and let $B=B(D)$. Then $B$ is a Banach algebra with the supremum norm on $D$. The spectrum of $B$, denoted by $S(B)$, is the set of nonzero complex homomorphisms of $B$. For $z \in D$ if we define $h_{z}(f)=f(z), f \in B$, then $h_{z} \in S(B)$. Hence we obtain a mapping $\rho: D \rightarrow S(B), \rho(z)=h_{z}$. To each $f \in B$ we associate a function $\hat{f}$ defined on $S(B)$ by defining $\hat{f}(h)=h(f)$. Since $\hat{f} \circ \rho=f$, the mapping $f \mapsto \hat{f}$ is one-to-one. We endow $S(B)$ with the weakest topology which makes $\hat{f}$ continuous. Then $S(B)$ is compact and the mapping $f \mapsto \hat{f}$ is an isometry of $B$ onto $\hat{B}=\{\hat{f}: f \in B\}$. Hence we may assume that $B$ is defined on $S(B)$. Let $f_{1}, \ldots, f_{n} \in B$. The joint spectrum of $f_{1}, \ldots, f_{n}$ is the set; $\sigma\left(f_{1}, \ldots, f_{n}\right)$ $=\left\{\left(f_{1}(h), \ldots, f_{n}(h)\right): h \in S(B)\right\}$. For given $f_{1}, \ldots, f_{n} \in B$ we define $\pi: S(B) \rightarrow C^{n}$ by $\pi(h)=\left(f_{1}(h), \ldots, f_{n}(h)\right)$, then $\pi$ is a continuous map. If $D$ is relatively compact in $C^{n}$ then the coordinate functions $z_{1}, \ldots, z_{n}$ belong to $B$ and $\pi S(B) \supset D$ since the point evaluation maps are in $S(B)$. Furthermore, since $S(B)$ is compact $\pi S(B) \supset \bar{D}$. Now we have the following theorem:

22. THEOREM. Let $D$ be a relatively compact region in $C^{n}$ with int $\bar{D}=D$. If $\pi S(B)=\bar{D}$ then $D$ is a region of bounded holomorphy.

Proof. If we assume that $D$ is not a region of bounded holomorphy, then every function $f \in B$ has an extension $f$ to a neighborhood $V$ of a boundary point $p$ of $D$. By Lemma $15, \tilde{f}(V) \subset \operatorname{cl}(f(D))$. Hence the extensions $\tilde{f}, f \in B$ are continuous with respect to the supnorm on $D$. Now take a point $z \in V-\bar{D}$, consider the point evaluation map $h_{z}, h_{z}(\tilde{f})=\tilde{f}(z)$ for all $f \in B$, then $h_{z} \in S(B)$ and $\pi\left(h_{z}\right)=z \in V-\bar{D}$, which is absurd.

We note that if int $\bar{D} \neq D$ then the theorem is false; consider

$$
D=\{z \in C: 0<|z|<1\}
$$

then $B(D)=B(D \cup\{0\})$ and $S(B)=\bar{D}$. But $D$ is not a domain of bounded holomorphy.

\section{REFERENCES}

1. R. Gunning and H. Rossi, Analytic functions of several complex variables, Prentice-Hall, Englewood Cliffs, N. J., 1965. MR 31 \#4927.

2. L. Hörmander, An introduction to complex analysis in several variables, Van Nostrand, Princeton, N. J., 1966. MR 34 \#2933.

3. F. Quigley, Lectures on several complex variables, Tulane University, New Orleans, La., $1964 / 65,1965 / 66$.

4. W. Rudin, Some theorems on bounded analytic functions, Trans. Amer. Math. Soc. 78 (1955), 333-342. MR 16, 685.

5. M. Shauck, Algebras of holomorphic functions in ringed spaces, Dissertation, Tulane University, New Orleans, La., 1966.

UNIVERSITY OF FLORIDA, Gainesville, Florida 32601 